\title{
Agiles Sprintlernen wirkt - aber warum? Theoriegeleitete Analyse der Wirkprinzipien eines Gestaltungsansatzes für arbeitsbezogene Kompetenzentwicklung
}

\author{
Joana Jungclaus ${ }^{1}$ (D) $\cdot$ Niclas Schaper ${ }^{2}$ \\ Angenommen: 2. Januar 2021 / Online publiziert: 19. Januar 2021 \\ (c) Der/die Autor(en) 2021
}

\section{Zusammenfassung}

Dieser Beitrag der Zeitschrift Gruppe. Interaktion. Organisation. (GIO) stellt eine theoriegeleitete Analyse der Wirkprinzipien des Agilen Sprintlernens vor. Agiles Sprintlernen ist ein neuartiger Gestaltungsansatz für arbeitsbezogene Kompetenzentwicklung. Die bisherige Forschung zeigt positive Ergebnisse hinsichtlich des erfolgreichen Kompetenzerwerbs, der Zufriedenheit mit dem Lernerfolg sowie der Bewertung und Akzeptanz der Lernform. Bislang wurde jedoch nicht durchgängig untersucht, wie und warum das agile Sprintlernen wirksam ist. Dem methodischen Ansatz der Programmevaluation folgend, wird analysiert, welche lerntheoretischen Konstrukte den Gestaltungsansatz im Besonderen kennzeichnen und inwieweit diese die Wirkweise des agilen Sprintlernens erklären können.

Die Ergebnisse zeigen, dass das agile Sprintlernen durch neun zentrale Gestaltungs- und Wirkungselemente gekennzeichnet ist: Zielorientierung und Zielverfolgung, Transparenz und Klarheit, Planung des Lernens, Eigenaktivität und Eigenverantwortung, Feedback, Selbstreflexion, Autonomie, Kompetenzerleben und soziale Eingebundenheit. Zur theoretischen Fundierung und Herleitung potenzieller Wirkmechanismen im agilen Sprintlernen werden mehrere lerntheoretische Bezugspunkte herangezogen - u. a. die Zielsetzungstheorie und die Theorie der sozialen Interdependenz. Die Ergebnisse werden abschließend diskutiert.

Schlüsselwörter Agiles Sprintlernen · Arbeitsbezogenes Lernen · Agiles Lernen · Programmevaluation · Theoretische Fundierung

\section{Agile Sprintlearning works-but why? Learning theoretical analysis of the effect mechanisms of a conceptual framework for work-related learning}

\begin{abstract}
This article in the journal Gruppe. Interaktion. Organisation. (GIO) portrays a theory-driven analysis of the effect mechanisms of Agile Sprintlearning. Agile Sprintlearning is a novel framework for work-related skills development. Former research shows positive results with regards to competency acquisition, satisfaction with the learning success as well as the assessment and acceptance of the learning format. So far, however, it has not been consistently studied how and why agile Sprintlearning is effective. Following the methodological approach of theory-driven evaluation, which learning-theoretical constructs characterize the conceptual framework in particular and in which way they can explain the effectiveness of agile Sprintlearning.
\end{abstract}

Joana Jungclaus

joana.jungclaus@znl-ulm.de

1 ZNL TransferZentrum für Neurowissenschaften und Lernen, Universität Ulm, Parkstr. 11, 89073 Ulm, Deutschland

2 Institut für Humanwissenschaften, Lehrstuhl für Arbeits- und Organisationspsychologie, Universität Paderborn, Warburger Straße 100, 33098 Paderborn, Deutschland 
The results show that agile Sprintlearning can be characterized by nine central design and effect elements: goal orientation and goal tracking, transparency, planning of learning, self-activity and personal responsibility, feedback, self-reflection, autonomy, competence experience and social relatedness. For their theoretical foundation and in order to derive potential effect mechanisms of agile Sprintlearning, several learning-theoretical reference points are used-including goal setting theory and social interdependence theory. The results of the learning theoretic substantiation are finally discussed.

Keywords Agile Sprintlearning - Work-related learning - Agile learning · Theory-driven evaluation · Theoretical foundation

\section{Einleitung}

Die Rahmenbedingungen der heutigen Arbeitswelt - u.a. dezentralisierte und teamorientierte Arbeits- und Organisationsstrukturen, Digitalisierung und verkürzte Innovationszyklen - haben vielschichtige Konsequenzen für Lernen und Kompetenzentwicklung in Organisationen. Sie resultieren vermehrt in steigenden Anforderungen an die berufliche Handlungskompetenz von Mitarbeitenden sowie deren Umgang mit neuem und vielfältigem Wissen (Schaper und Sonntag 2007). Außerdem sind die Umgebungsbedingungen der heutigen Arbeitswelt zunehmend instabil, unvorhersehbar und schnelllebig. Mit Blick auf den Erhalt der Wettbewerbsfähigkeit werden Flexibilität und Veränderungsfähigkeit daher immer bedeutsamer (von Ameln und Wimmer 2016), was die Ansprüche an Lernen und Kompetenzentwicklung ebenfalls erhöht. All diese Anforderungen verlangen Organisationen ein hohes $\mathrm{Maß}$ an kontinuierlicher, selbstgesteuerter und flexibler Kompetenzentwicklung ab (Korge et al. 2018). Um effektiv zu sein, ist dabei jedoch auch gezielte instruktionale Unterstützung erforderlich (Schaper 2000).

Ein neuartiger Gestaltungsansatz für arbeitsbezogenes Lernen, der diesen Herausforderungen begegnen soll, ist das agile Sprintlernen (Jungclaus et al. 2019; Korge et al. 2018). Ziel ist der bedarfsorientierte und arbeitsintegrierte Erwerb und Ausbau beruflicher Handlungskompetenzen von Organisationsmitgliedern. Der Ansatz wurde im Rahmen des Forschungsvorhabens in MEDIAS res von den Forschungsinstituten Fraunhofer IAO und dem ZNL TransferZentrum für Neurowissenschaften und Lernen über einen Zeitraum von mehr als zwei Jahren in vier Unternehmen unterschiedlicher Größen und Branchen entwickelt, erprobt und evaluiert. Die Evaluationsstudien zum Einsatz des agilen Sprintlernens zeigen positive Ergebnisse hinsichtlich eines erfolgreichen Kompetenzerwerbs, der Zufriedenheit mit dem Lernerfolg sowie der Bewertung und Akzeptanz der Lernform. Unter Beachtung bestimmter qualitätsrelevanter Umsetzungsaspekte stellt agiles Sprintlernen einen wirksamen Rahmen für arbeitsbezogene Kompetenzentwicklung dar (Bauer et al. under review; Jungclaus 2021).

Aus empirischer Sicht ist bislang jedoch nicht bzw. nur unzureichend geklärt, wie und warum das agile Sprintler- nen wirksam ist. Ziel dieses Artikels ist daher, potenzielle Wirkmechanismen des Lernansatzes zu identifizieren und damit im Sinne der Programmevaluation (Chen 1990, 2012) einen ersten wichtigen Beitrag zur umfassenden, insbesondere theoriegeleiteten Evaluation des agilen Sprintlernens zu leisten. Dafür wird analysiert, welche lerntheoretischen Konstrukte den Gestaltungsansatz im Besonderen kennzeichnen und inwieweit diese die Wirkweise des agilen Sprintlernens erklären können.

\section{Agiles Sprintlernen und Stand der Forschung}

Bevor das methodische Vorgehen zur Identifikation der lerntheoretischen Konstrukte und potenziellen Wirkmechanismen dargestellt wird (s. Abschn. 3), wird zunächst ein Überblick über das Sprintlernen ${ }^{1}$, die zu Grunde liegenden lerntheoretischen Axiome bei dessen Entwicklung sowie den aktuellen Stand der Forschung gegeben.

\subsection{Der Gestaltungsansatz Agiles Sprintlernen}

Wie bereits erwähnt, müssen Mitarbeitende und Organisationseinheiten zunehmend eigenverantwortlich und kontinuierlich lernen, um den wachsenden Anforderungen und der immer höheren Veränderungsgeschwindigkeit gerecht zu werden. Im Sinne der Kompetenzstufen nach Rauner et al. (2007) sind dafür vermehrt prozessuale Kompetenzen (d.h. umfassende Fähigkeiten zur situationsabhängigen Bewertung und Handlung mit Bezügen zu Arbeitsprozessen) oder ganzheitliche Gestaltungskompetenz (d.h. Fähigkeiten zur Deutung und Bewältigung komplexer Situationen unter divergierenden Anforderungen) erforderlich. Sprintlernen zielt darauf ab, berufliche Handlungskompetenzen auf einer mindestens prozessualen Kompetenzstufe aufzubauen. Mittels klassischer Lernformen, die oftmals eine hohe Distanz zum Arbeitsort und wenig Anwendungsbezug haben, erwerben Mitarbeitende Kompetenzen oftmals nur auf

\footnotetext{
Aus Gründen der besseren Lesbarkeit steht im Text Sprintlernen stellvertretend für agiles Sprintlernen.
} 
funktionaler Stufe (d.h. Fachkenntnisse und Fertigkeiten ohne Zusammenhangsbezüge und Deutungen).

Darüber hinaus verfolgt Sprintlernen das Ziel, die Kompetenzentwicklung selbst flexibel, bedarfsorientiert, arbeitsnah und kontinuierlich zu gestalten.

Die Lernanlässe für das Sprintlernen ergeben sich direkt aus der Arbeit bzw. den zukünftigen Anforderungen an das Arbeitshandeln von Organisationsmitgliedern. Die Lerninhalte können demnach Handlungskompetenzen unterschiedlichster Art sein - bspw. Fachkompetenzen, die direkt zur Ausführung des Arbeitsablaufs notwendig sind (F\&E-Prozesse, Kundenberatung, Bedienung von Datenbanken im Arbeitskontext u. Ä.). Neben den fachlichen Anforderungen werden kontextabhängig auch andere relevante Kompetenzarten wie Methoden- oder Personalkompetenzen im jeweiligen Sprintlernen integriert (bspw. Kommunikation zwischen Berufsgruppen im relevanten Arbeitsprozess, Projektmanagement), um die beruflichen Handlungskompetenzen ganzheitlich zu erwerben.

Als didaktisches Rahmenkonzept definiert das Sprintlernen daher spezifische Rollen und Abläufe bei der Lernprozessgestaltung und ist anpassbar auf verschiedene Lerninhalte, Kontexte und Zielgruppen in Organisationen.

\subsubsection{Rollen und Ablauf}

Die Rollen und Ablaufelemente sind themenbezogen und unternehmensspezifisch auszugestalten. Es gibt vier Rollen bzw. Funktionsträger im Sprintlernen: Lernende bzw. Lernteam, das aus max. acht Lernenden besteht, Fachexperte ${ }^{2}$ (einer oder mehrere), Kunde und Sprintbegleiter.

Die Mitarbeitenden verpflichten sich als Lernteam einem gemeinsamen Lernauftrag zum Aufbau relevanter beruflicher Kompetenzen. Der Kunde - meist die Führungskraft der Abteilung bzw. des Bereiches, in dem Kompetenzen $\mathrm{zu}$ erwerben oder zu erweitern sind - ist der „Auftraggeber" des Lernens und gibt die dafür benötigten Ressourcen frei (insbes. zeitliche Ressourcen für das Lernen). Auch die Mitarbeitenden selbst können Kompetenzbedarfe einbringen. In diesem Fall gibt der Kunde das Lernthema frei und stellt die entsprechenden Ressourcen dafür bereit. Fachlich wird der Lernauftrag durch den Fachexperten begleitet, der die fachlichen Anforderungen genauer spezifiziert, als mögliche Wissensquelle dient und die (Lern-)Ergebnisse des Lernteams abnimmt. Je nach Lerninhalt und -gegenstand kann der Fachexperte intern oder extern und durch eine oder mehrere Personen besetzt sein. Der Sprintbegleiter ist fachfremd und übernimmt die Funktion eines Lernprozess-

\footnotetext{
${ }^{2}$ Aus Gründen der besseren Lesbarkeit wird auf die gleichzeitige Verwendung von männlicher und weiblicher Sprachformen verzichtet. Sämtliche Personenbezeichnungen gelten gleichermaßen für beiderlei Geschlechter.
}

begleiters. Er unterstützt das Lernteam methodisch-didaktisch bei der Erfüllung des gemeinsamen Lernauftrags und organisiert den gesamten Prozess. Er spielt außerdem eine wichtige Rolle in der Vor- und Nachbereitung des Sprintlernens - z. B. wenn es darum geht, den Fachexperten bei der Analyse der Kompetenzbedarfe und der themenspezifischen Definition der sog. Lernaufgaben und Akzeptanzkriterien zu unterstützen.

Die Ablaufelemente umfassen eine Vorbereitungs-, eine Durchführungs- und eine Nachbereitungsphase, wobei die Durchführung aus wiederkehrenden Zyklen von Planungstreffen, Lernsprint, Ergebnisabnahme und Teambesprechung besteht. Abb. 1 zeigt den Ablauf des Sprintlernens mit den zugehörigen teilnehmenden Rollenträgern im Überblick.

Ist ein Kompetenzbedarf geeignet für Sprintlernen, analysieren Sprintbegleiter und Fachexperte im Rahmen der Vorbereitungsphase benötigte Teilkompetenzen des Lernthemas (z.B. Aspekte, die neben Fachkenntnissen für die Erfüllung eines bestimmten Arbeitsprozesses notwendig sind). Daraufhin definiert der Fachexperte mit Unterstützung des Sprintbegleiters sog. Lernaufgaben, die das Lernteam in der Durchführungsphase selbstgesteuert bearbeitet (z.B. entlang des Arbeitsablaufs, pro Prozessschritt ein oder mehrere Lernaufgaben mit authentischen Anwendungssituationen). $\mathrm{Zu}$ jeder Lernaufgabe werden „Akzeptanzkriterien“ definiert, die das Ziel der Aufgabe konkretisieren und anhand möglichst messbarer Kriterien beschreiben, ab wann eine Lernaufgabe als erfüllt gilt. Hilfreiche Dokumentationen, Wissensquellen oder Ansprechpartner werden ebenfalls aufgeführt, sodass das Lernteam während der Bearbeitung darauf zurückgreifen kann.

Die Durchführung der Lernprozesse verläuft iterativ mit definierten und wiederkehrenden Bestandteilen zu Lernplanung, Kompetenzaufbau, Ergebnisabnahme und Prozessreflexion. Jeder Abschnitt beginnt mit einem Planungstreffen, das der genaueren Auseinandersetzung mit den Anforderungen (d.h. den Lernaufgaben und Akzeptanzkriterien) und der Festlegung einer Lernstrategie für den kommenden Lernsprint dient. Das Lernteam hat die Möglichkeit, Rückfragen an den Fachexperten zu stellen und plant, wie viele Lernaufgaben im anstehenden Lernsprint mit welcher Strategie bearbeitet werden (z.B. Bearbeitung im Team oder einzeln, Einbezug des Fachexperten).

Die Lernsprints, in der die Aufgaben selbstgesteuert bearbeitet werden, können unterschiedlich lang sein. Bei einem Lernen in Vollzeit kann ein Lernsprint bspw. einen Tag dauern - bei arbeitsintegriertem Sprintlernen kann ein Lernsprint eine Länge von ein bis vier Wochen haben, in denen die Teammitglieder einen vorher definierten und freigegebenen Lernaufwand von wenigen Stunden während der Arbeitszeit unterbringen. Sprintbegleiter und Fachexperte 
Abb. 1 Ablaufelemente des agilen Sprintlernens mit dazugehöriger Zielsetzung und teilnehmenden Rollenträgern (entnommen aus Jungclaus et al. 2019)

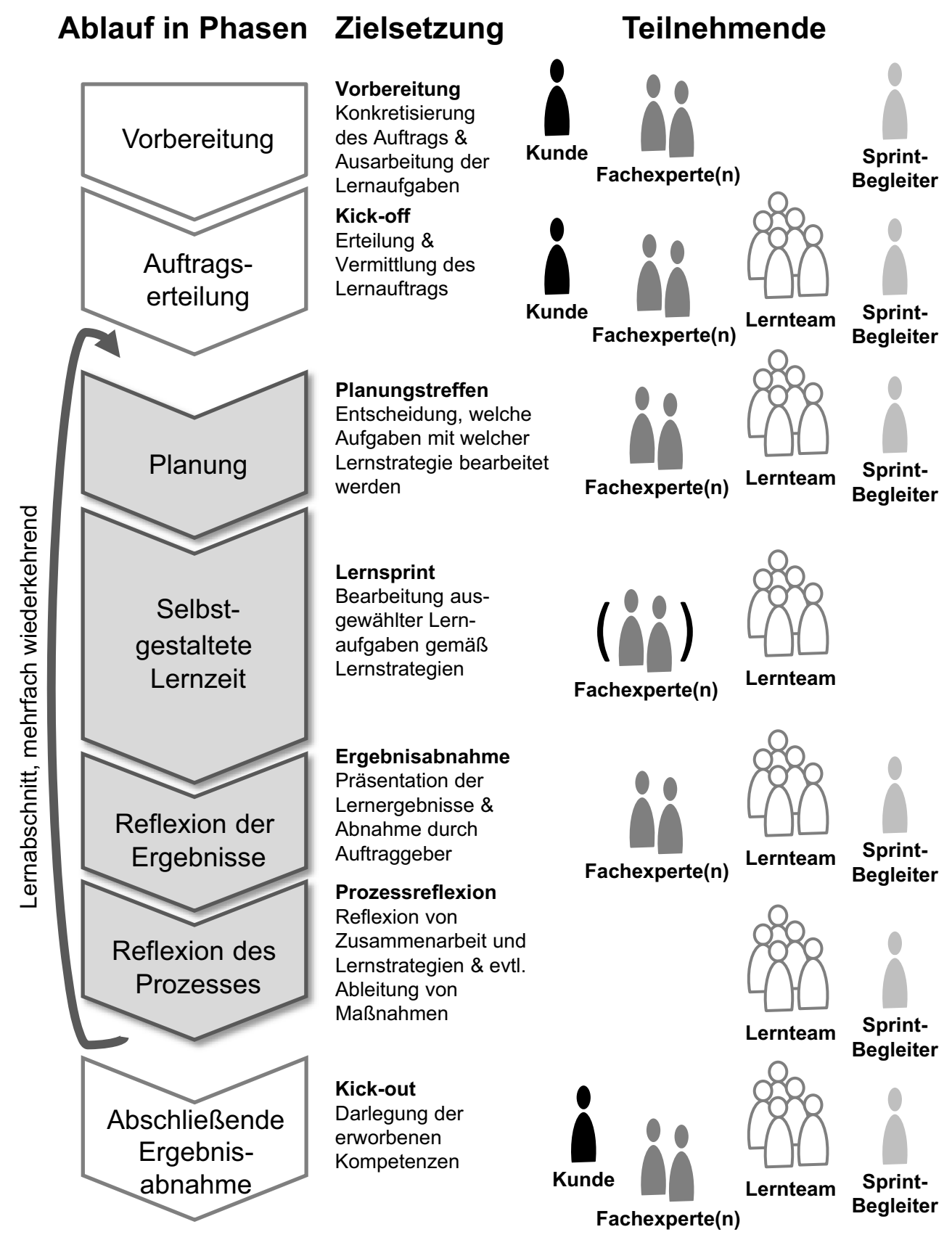

stehen während der selbstgestalteten Lernzeit auf Anfrage zur Verfügung.

Am Ende eines jeden Lernsprints stehen zwei Treffen: Ergebnisabnahme und Teambesprechung. Im Rahmen der Ergebnisabnahme werden die Lernaufgaben, die im letzten Lernsprint bearbeitet wurden, durch das Lernteam vorgestellt. Der Fachexperte kann Rückfragen stellen und fachliche Ergänzungen geben. Er entscheidet, inwieweit das Lernteam alle Akzeptanzkriterien einer Aufgabe erfüllt hat und nimmt die Ergebnisse ab. Ist eine Lernaufgabe nicht oder nur teilweise erfüllt, erläutert der Fachexperte, was genau fehlt und das Team bearbeitet die entsprechende Lern- aufgabe im kommenden Lernsprint erneut bzw. vervollständigt sie. In der Teambesprechung reflektiert das Lernteam den Lernprozess zusammen mit dem Sprintbegleiter und leitet ggfs. Anpassungen für den folgenden Lernsprint ab, die bei der darauffolgenden Planung der Lernstrategie berücksichtigt werden. Die Kompetenzen werden sukzessive erworben - beim arbeitsintegrierten Sprintlernen erstreckt sich der Lernprozess über mehrere Wochen und weist eine hohe zeitliche und räumliche Nähe zur Arbeit auf.

Nach Abschluss der geplanten Lernsprints besteht die Möglichkeit, Teilaufgaben in nachgelagerten Lernsprints zu wiederholen oder zu vertiefen. Wenn sich die Anfor- 
derungen an die Arbeit nach Beendigung des Sprintlernens grundlegend ändern oder erweitern (z.B. aufgrund technischer Weiterentwicklungen o. Ä.), können diese Inhalte in neu aufgesetzten Lernsprints bearbeitet werden.

\subsubsection{Vorgehen zur Entwicklung und Erprobung}

Wie in Abschn. 2.1 beschrieben, war der Ausgangspunkt bei der Entwicklung des Sprintlernens die Zielsetzung, arbeitsbezogene Kompetenzentwicklung bedarfsorientierter, flexibler und kontinuierlicher zu gestalten. Es wurde angenommen, dass agile Projektmanagementmethoden - insbesondere Scrum (Roock und Wolf 2015) - ein geeignetes Rahmenwerk für solche Lernprozesse liefern können (Korge et al. 2018). Zu Beginn der Entwicklung wurden daher jene Elemente aus Scrum bestimmt, die unter Gesichtspunkten des gelingenden Lernens als förderlich für Lernkonzepte zum Erwerb beruflicher Handlungskompetenzen erachtet wurden. Diese wurden auf den beschriebenen Lernkontext übertragen und angepasst (bspw. Rollen und Abläufe aus Scrum). Weiterhin wurde das Sprintlernen um eigens entwickelte Elemente ergänzt, die sich vor allem aus der Übertragung bereits gesicherter empirischer Erkenntnisse zur Gestaltung aktivierenden arbeitsbezogenen Lernens ergeben (s. Abschn. 2.2).

Während der über zweijährigen Erprobungsphase wurden insgesamt 19 Praxisumsetzungen zu unterschiedlichen Lernthemen bei vier Unternehmenspartnern durchgeführt und durch das Forschungsteam begleitet. Die einzelnen Gestaltungselemente wurden dabei iterativ angepasst und optimiert. Die formative Evaluation lieferte direkte Hinweise zur Verbesserung des Lernansatzes, sodass bestehende Elemente konkretisiert, neue Elemente ergänzt oder nicht zielführende Elemente eliminiert wurden. Auch dabei waren lerntheoretische Axiome handlungsleitend (s. Abschn. 2.2).

\subsection{Lerntheoretische Axiome bei der Entwicklung des agilen Sprintlernens}

Wie in Abschn. 2.1.2 angesprochen, liegen dem Lernkonzept des Sprintlernens eine Reihe von axiomatischen Grundsätzen zu Grunde. Damit sind verschiedene Vorannahmen bzw. lerntheoretische Postulate und Prämissen für effektives Lernen gemeint, auf deren Grundlage das Sprintlernen konzipiert wurde. Die lerntheoretischen Axiome leiten sich aus der aktuellen Befundlage zum wirksamen Kompetenzerwerb in der Arbeit ab. Sie waren sowohl für die Entwicklung als auch für die Optimierung des Sprintlernens handlungsleitend (s. Abschn. 2.1.2).

\subsubsection{Axiom 1: Lernverständnis des gemäßigten Konstruktivismus (Kirschner et al. 2006; Mietzel 2017; Reinmann und Mandl 2006)}

Im Sprintlernen wird Lernen als ein aktiver und selbstgesteuerter Prozess mit eigenen Konstruktionsleistungen angesehen, bei dem jedoch ein Mindestma $\beta$ an instruktionalen Strukturen notwendig ist. Insbesondere während der selbstgestalteten Lernzeit gibt es aktive Aneignungsprozesse, bei denen die Lernaufgaben selbstgesteuert bearbeitet werden. Die Lernteams wählen ihre Lernstrategien und -zeiten eigenständig und fertigen bei Bedarf eigene Ergebnisdokumentationen an. Die themenspezifische Vorbereitungsphase und die anwendungsbezogenen Lernaufgaben führen zu situativen und kontextbezogenen Lernprozessen (s. a. Axiom 2). Die Haltung des Sprintbegleiters wurde ebenfalls in Anlehnung an das konstruktivistische Lernverständnis hin orientiert, weil dieser in der Haltung eines Lernprozessbegleiters agiert. Dadurch, dass im Team gelernt wird und dass Lern- und Teamprozesse regelmäßig mit Unterstützung des Sprintbegleiters reflektiert werden, wird das Lernen als emotionaler und sozialer Prozess angesehen (s. a. Axiom 5). Die Summe der Lernaufgaben beinhaltet verschiedene relevante Anwendungssituationen des zu erwerbenden Wissens (multiple Kontexte). Durch die Lernaufgaben selbst und/ oder durch die Bearbeitung als Team und durch die Rückmeldung des Fachexperten wird die relevante Problemstellung außerdem aus verschiedenen Perspektiven betrachtet. Die teils heterogene Zusammensetzung der Lernteams unterstützt diesen Aspekt ebenfalls.

Instruktionale Anteile spiegeln sich in vordefinierten Lernaufgaben und -zielen wider, weil der umfassende Lernauftrag in einzelne Lernaufgaben heruntergebrochen und damit die Lerninhalte in handhabbare Lerneinheiten aufgeteilt wird. Lernende können bei Bedarf zwar eigens definierte Lernaufgaben einbringen - der überwiegende Teil der Lernaufgaben und damit auch der Lernziele ist jedoch vorgegeben. Weitere instruktionale Elemente sind die Ergebnisabnahmen sowie die bedarfsbezogene Unterstützung durch Fachexperten und Sprintbegleiter während des Lernprozesses. In den Ergebnisabnahmen kann der Fachexperte korrigierend einwirken, indem er fachliche Ergänzungen vornimmt oder auf fehlende Aspekte des Lernergebnisses hinweist. Während der selbstgestalteten Lernzeit erhält das Lernteam auf Anfrage Unterstützung durch Fachexperten bzw. Sprintbegleiter, wodurch das Ausmaß an Instruktion ggf. zunimmt und das Ausmaß an Selbststeuerung abnimmt.

\subsubsection{Axiom 2: Situiertes Lernens (Schaper 2007, 2019a)}

Die Analyse des Lernthemas in seinem Anwendungskontext und die anwendungsbezogenen Lernaufgaben, die sich 
auf den spezifischen Arbeitskontext der Lernteammitglieder beziehen, gewährleisten einen anwendungsbezogenen und situierten Wissens- und Fähigkeitserwerb. Durch die authentischen Aufgaben und das hohe Maß an Selbststeuerung beinhalten die Lernprozesse aktive Konstruktionsleistungen. Ein weiteres ,,situiertes“ Gestaltungsprinzip des Sprintlernens ist, dass der Arbeitsort als Lernort dient und der Lernprozess damit in die Arbeit eingebettet ist - sowohl räumlich als auch zeitlich. Gleichzeitig findet damit das Lernen auch in einem authentischen sozialen Umfeld statt.

\subsubsection{Axiom 3: Handlungsorientiertes Lernen in Anlehnung an die Handlungsregulationstheorie (Frese und Zapf 1994; Hacker 2005)}

Mit Bezug auf die Handlungsregulationstheorie zielt das Sprintlernen auf den Erwerb ganzheitlicher aufgabenbezogener Handlungskompetenzen. Der Gestaltungsansatz berücksichtigt in diesem Zusammenhang die zentralen Elemente bzw. Aspekte der Handlungssteuerung, die in den verschiedenen Phasen des Handelns im Vordergrund stehen (Phasen bzw. Prozesse der Zielbildung, Orientierung, Planung, Ausführung, Kontrolle und des Feedbacks). Die Lernaufgaben mit Akzeptanzkriterien stellen konkrete Ziele dar. Die Planungstreffen dienen zur Orientierung über den Lern- bzw. Handlungsprozess und zur Formulierung einer (Lern-)Strategie für die Bearbeitung der Lernaufgaben. Auf dieser Grundlage führen die Lernteams die Handlungen und Aufgaben weitgehend selbstständig aus und kontrollieren dabei anhand der Akzeptanzkriterien den Grad der Zielerreichung bzw. Erfüllung der Lernaufgaben. Die Reflexion der erzielten Ergebnisse und des vollzogenen Lernprozesses erfolgt durch die beiden separaten Feedbackeinheiten, mit denen jeder Lernsprint abgeschlossen wird. Durch den Sprintbegleiter wird diese Struktur sichergestellt und methodische Unterstützung geleistet.

Die Bezüge der Kompetenzen zu den relevanten Arbeitssituationen und -kontexten wird durch den hohen Arbeits- und Kontextbezug der Lernaufgaben gewährleistet, wodurch auch relevantes tätigkeits- und aufgabenspezifisches Wissen erworben wird.

Im Rahmen des Sprintlernens wird vor allem auf das Konzept der vollständigen Handlung bzw. der zugehörigen Handlungsphasen Bezug genommen, während weitere Theorieelemente der Handlungsregulationstheorie, z. B. die Unterscheidung von verschiedenen Ebenen der Handlungsregulation (Schaper 2019b), bei der Konzeption des Sprintlernens nicht berücksichtigt werden.

\subsubsection{Axiom 4: Selbstreguliertes Lernen in Anlehnung an den Ansatz des self-regulated learning (Zimmerman 2008)}

Analog zu den Phasen der Handlungssteuerung (s. Axiom 3) werden die Phasen des selbstregulierten Lernens im Sprintlernen explizit berücksichtigt und angesprochen (Vorschauphase, Lern- und Leistungsphase, Selbstreflexionsphase). Das Lernteam steht als aktiv handelnd im Mittelpunkt. Es setzt sich vor jedem Lernsprint mit den anstehenden Lernaufgaben auseinander und plant seine Strategien. Dabei wird auch ausgetauscht, welche Aspekte den Teammitgliedern in der Zusammenarbeit und für motiviertes Lernen wichtig sind. Während der selbstgestalteten Lernzeit sind Prozesse wie Fokussierung der Aufmerksamkeit oder Zeitmanagement von starker Bedeutung, weil das Lernen parallel zur Arbeit stattfindet. Die Komponente der Selbstreflexion wird durch die Feedbackeinheiten am Ende jeden Lernsprints sowie Anregungen des Sprintbegleiters gefördert. In der Prozessreflexion leitet das Lernteam ggf. Anpassungen für den nächsten Lernsprint ab. Der iterative Charakter des Vorgehens beim Teamlernen ermöglicht damit kurzzyklische Soll-Ist-Abgleiche und adaptive Zielverfolgung, welche kennzeichnend für selbstreguliertes Lernen sind. Sprintlernen bietet damit einen Rahmen, der selbstreguliertes Lernen aufgrund seiner Struktur explizit unterstützt. Darüber hinaus können Lernende durch das Durchlaufen der Sprintstruktur ihre eigene Fähigkeit zur Selbstregulation trainieren und ggf. auch für andere Aufgaben und Tätigkeitsanforderungen davon profitieren.

\subsubsection{Axiom 5: Kooperatives Lernen (Hattie 2008; Reinmann und Mandl 2006)}

Durch das Lernen in Teams und die Lernaufgaben, die das soziale Gefüge in der Anwendungssituation des Wissens berücksichtigen, werden die Lernprozesse im Sprintlernen in ihrer jeweils spezifischen sozialen Umwelt ausgeführt und sind in diese eingebettet. Lernen findet in Interaktion statt, Kooperation ist ein fester Bestandteil des Lernprozesses und Problemlösen in Gruppen wird ermöglicht. Die Lernteams können sich aus Teammitgliedern mit sehr heterogenen Hintergründen bzgl. Profession, Berufsgruppe, Hierarchiestufe u. Ä. zusammensetzen. So besteht ein Lernteam beispielsweise aus Ingenieuren und Mechanikern, die im betrieblichen Kontext am selben Arbeitsprozess beteiligt sind und damit interdependent an einem übergeordneten Arbeitsauftrag arbeiten. Dies spiegelt sich auch in dem gemeinsamen Lernauftrag für das Sprintlernen. Die Lernteammitglieder sind vor diesem Hintergrund in der Lage, sich auf gemeinsame Lernziele zu verpflichten und die (Lern-)Ergebnisse als Team abnehmen zu lassen. Insbesondere bei stark ausgeprägter Heterogenität im Lernteam kön- 
nen sich die Gruppenmitglieder in ihrem Wissen und ihren Fähigkeiten ergänzen.

\subsubsection{Axiom 6: Selbstbestimmtes Lernen in Anlehnung an die Selbstbestimmungstheorie der Motivation (Deci und Ryan 2000)}

Im Sprintlernen soll das Lernen mit möglichst selbstbestimmten Formen der Motivation erfolgen. Die Lernthemen werden zumeist unternehmensseitig angestoßen, weshalb nicht per se von intrinsischer Lernmotivation bei den Lernteammitgliedern ausgegangen werden kann. Das didaktische Rahmenkonzept umfasst jedoch eine explizite Vorbereitungsphase, in der das Lernthema auf Eignung für Sprintlernen geprüft wird. Ein Kriterium für die Eignung ist, dass das Thema sowohl für Unternehmen als auch für die Lernenden selbst von hoher Relevanz ist. Darüber hinaus werden die Ressourcen für das Lernen explizit durch den Kunden, d.h. den Auftraggeber des Lernteams freigegeben, um Zielkonflikte für die Lernteams zu vermeiden. Dazu zählen die Lernzeiten der Lernteammitglieder, aber auch die zeitlichen Aufwände der Fachexperten und Sprintbegleiter. Obwohl der Lernprozess meistens aufgrund eines äußeren Anreizes initiiert wird, können sich die Lernenden mit hoher Wahrscheinlichkeit mit dem Ziel identifizieren, weil sich die Lerngegenstände aus den individuellen aktuellen bzw. zukünftigen Anforderungen an die eigenen Arbeitsaufgaben ergeben. Je nachdem, ob und wie stark sich andere Ziele der Lernenden - bspw. aktuelle Projektziele aus dem Arbeitshandeln - konfliktär zu den Lernzielen verhalten, liegen im Sprintlernen daher die Motivationstypen identifizierte Regulation (Zielkonflikte vorhanden) oder integrierte Regulation (keine Konflikte mit anderen Zielen) vor, was selbstbestimmtem Lernen entspricht.

\subsection{Stand der Evaluierung}

Neben der in Abschn. 2.1.2 erwähnten formativen Evaluation, wurden die Praxisumsetzungen des Sprintlernens auch summativ evaluiert (Bauer et al. under review). Im Fokus standen dabei die Fragen, ob das Sprintlernen im Sinne eines erfolgreichen Kompetenzerwerbs wirksam ist und wie beteiligte Rollenträger aus der Praxis (Lernteams, Fachexperten und Sprintbegleiter) das Sprintlernen als Lernform bewerten.

Gegenstand der Analysen waren die 19 durchgeführten Praxisumsetzungen bei den vier Unternehmenspartnern des Forschungsvorhabens in MEDIAS res. Die Unternehmen stammen aus verschiedenen Branchen: Industrie, Finanzwirtschaft, IT- und Pharma-Dienstleistung. Die Lernthemen der Erprobungen umfassten die fachgerechte Bedienung von Hardware, Software oder Datenbanken im jeweiligen Arbeitsprozess und -kontext, technische Bewertungen,
Programmierung, aber auch Kompetenzbedarfe in den Bereichen Gesprächsführung und Change-Management/ Unternehmensstrategien. Das Forschungsteam begleitete die Umsetzungen vor Ort und beobachtete und dokumentierte die Prozesse systematisch. Diese Prozessdokumentation diente neben formativen Evaluationszwecken auch als Datenquelle und Interpretationshilfe für die summative Evaluation. Außerdem wurden die beteiligten Rollenträger aus den Praxisumsetzungen nach Abschluss des Sprintlernens mittels Fragebogen oder in Form eines standardisierten Evaluationsinterviews zu ihrem Erleben und zu Aspekten der Wirksamkeit und Bewertung des Sprintlernens befragt.

Im Rahmen der Auswertung wurde zunächst überprüft, inwieweit bei den Erprobungen tatsächlich von einer konzeptgetreuen Umsetzung des didaktischen Rahmenkonzepts agiles Sprintlernen ausgegangen werden kann. Dazu wurde ein Kriterienkatalog herangezogen, der die Gestaltungselemente mit ihrer Funktion und ihren Minimalaspekten einer konzeptgetreuen Umsetzung enthält (siehe Bauer et al. under review). Nach dieser Prüfung verblieben 15 Praxisumsetzungen in der Stichprobe für die vertiefenden Analysen.

Die Ergebnisse der summativen Evaluation zeigen, dass berufliche Handlungskompetenzen mittels Sprintlernen erfolgreich erworben werden können. Der Kompetenzzugewinn ist dem Lernkonzept insofern immanent, als dass die Fachexperten in den Ergebnisabnahmen anhand der definierten Akzeptanzkriterien prüfen, inwieweit das Lernteam (Teil-)Kompetenzen erfolgreich aufgebaut hat. Nicht oder nur teilweise erfüllte Lernaufgaben werden im darauffolgenden Lernsprint nachgeholt. Die Prozessdokumentation zeigt, dass die Summe aller Lernaufgaben am Ende jeder Praxisumsetzung erfolgreich bearbeitet und abgenommen wurden. Dazu muss einschränkend erwähnt werden, dass dies zwar eine „objektivierte Betrachtungsform“ für den Kompetenzzugewinn des Lernteams ist, es wurden jedoch keine themenbezogenen Leistungstests o. ä. zur Messung des Kompetenzzugewinns bei den Lernenden durchgeführt. Die positive Bewertung des Kompetenzerwerbs stützt sich also letztlich auf die (subjektive) Einschätzung des jeweiligen Fachexperten, welche wiederum auf den vorab definierten fachlichen Akzeptanzkriterien basiert. Zusätzlich wurde bei einer Teilstichprobe die subjektive Zufriedenheit der Lernteammitglieder mit ihrem Lernerfolg standardisiert erhoben. Die Ergebnisse sind sehr positiv (Bauer et al. under review).

Für die große Mehrheit der Erprobungen zeigen sich außerdem positive Ergebnisse hinsichtlich der Bewertung der Lernform und ihrer Akzeptanz. Im Mittel bewerten die beteiligten Rollenträger das Sprintlernen mit 7,51 von 10 Punkten (auf einer 10-stufigen Skala mit 1 Punkt als negativster und 10 Punkten als positivster Bewertung der Lernform). Sieben der 15 Praxisumsetzungen wurden mit 8,4 bis 9,4 Punkten im Mittel bewertet. Die drei am nega- 
tivsten bewerteten Erprobungen erhielten zwischen 4,8 und 6,2 von 10 Punkten im Mittel.

Die Befragten begründeten ihre Punktewertung im offenen Antwortformat. Als Begründungen für eine positive Bewertung der Lernform Sprintlernen wurden u.a. der erfolgreiche Kompetenzerwerb, austauschintensives Lernen im Team, aktives und selbstgesteuertes sowie kontextgebundenes Lernen angeführt. Negativ konnotierte Begründungen wurden lediglich in den Praxisumsetzungen genannt, die vergleichsweise geringe Punktzahlen erhielten. Sie umfassten u. a. einen mangelnden Lernzugewinn, aber auch umsetzungsrelevante Aspekte, die in den positiv bewerteten Praxisumsetzungen als positive Begründungen herangezogen wurden. Beispielsweise wurde die Ergebnisabnahme in positiv bewerteten Umsetzungen (d.h. von der Mehrzahl der Befragten) als sehr hilfreiches und strukturgebendes Element angeführt, da hierdurch eine Deadline für das Lernteam vorgegeben war und weil dieses Element wertvollen fachlichen Austausch und Feedback beinhaltete und den Lernfortschritt sichtbar machte. In den moderat bewerteten Praxisumsetzungen wurde die Ergebnisabnahme mitunter als negatives Element erlebt, das zu Termindruck führe und wenig Mehrwert hätte.

Die Unterschiedlichkeit zwischen sehr positiv und eher positiv oder moderat bewerteten Praxisumsetzungen lässt sich auf verschiedene Aspekte zurückzuführen: So scheint z.B. die Auswahl des Lernthemas besonders bedeutsam. Das Lernthema sollte hinreichend komplex sein (mind. prozessuale Kompetenzstufe) und aus Sicht der Lernenden als besonders relevant für ihre Arbeit wahrgenommen werden, damit das Sprintlernen positiv bewertet wird. Besonders positive Bewertungen gingen darüber hinaus mit einem sehr positiven Aufwand-Nutzen-Verhältnis des Sprintlernens aus Sicht der Befragten einher.

Die Ergebnisse zeigen, dass es stark auf die jeweilige Ausgestaltung der Rollen, Ablaufelemente und Instrumente des Sprintlernens in einem bestimmten Kontext ankommt entscheidend ist also das wie, wenn es um den wirksamen Einsatz des didaktischen Rahmenkonzepts geht (Bauer et al. under review). Die enge Begleitung der Praxisumsetzungen und die systematische Prozessdokumentation ermöglichten eine Identifikation und Analyse von umsetzungsbezogenen qualitätsrelevanten Aspekten im Sprintlernen. Hierzu zählen neben der bereits erwähnten Auswahl geeigneter Lernthemen bspw. die Haltung und eine ausgeprägte Situationskompetenz des Sprintbegleiters, eine hohe Qualität der Lernaufgaben und die Gestaltung eines positiven AufwandNutzen-Verhältnisses innerhalb der einzelnen Meetings im Lernprozess. Situationskompetenz bezieht sich in diesem Zusammenhang darauf, dass der Sprintbegleiter auf verschiedenste Situationen im Lernen flexibel reagieren kann, team- und themenbezogene Anpassungen vornimmt (z.B. Ausmaß der Unterstützung eines Lernteams während eines
Lernsprints) und den Gestaltungsraum, den das didaktische Rahmenkonzept lässt, auf positive Weise - d.h. im Sinne des erfolgreichen Kompetenzerwerbs - nutzt. Für einen ausführlichen Überblick der qualitätsrelevanten Aspekte auf der Umsetzungsebene siehe auch Jungclaus (2021).

Alles in allem zeigt sich, dass sich das Sprintlernen in unterschiedlichsten Kontexten und verschiedenen Themenbereichen als wirksamer Gestaltungsrahmen für Kompetenzentwicklung erwiesen hat. In diesem Zusammenhang wurde insbesondere die Bedeutung und wirkungsvolle Ausgestaltung der einzelnen Elemente analysiert. Auf Basis der bisherigen Entwicklungsarbeiten und Studien können jedoch keine Aussagen zur konkreten Wirkweise des Sprintlernens gemacht werden.

\section{Theoriegeleitete Evaluation und Herleitung der Forschungsfragen}

An der in Abschn. 2.3 beschriebenen offenen Frage setzt der Ansatz der theoriegeleiteten Evaluation an (Chen 1990, 2012). Chen argumentiert, dass rein methodenorientierte Evaluationsansätze das Erreichen von Zielen und Ergebnissen von Interventionen betrachten, dabei jedoch oftmals nicht sensibel für den organisationalen Kontext sind, die (vollständige) theoretische Basis einer Intervention vernachlässigen und die Wirkmechanismen oder Kausalitäten hinter den beobachteten Effekten nicht erforschen. Sein konzeptueller Ansatz der Programmtheorie (Chen 1990, 2012) liefert einen Orientierungsrahmen für ganzheitliche Evaluationen von Interventionen, die auch untersuchen, warum und wie ein bestimmtes Interventionsprogramm wirkt. Auf diese Weise können sowohl Implikationen für die Weiterentwicklung der untersuchten Intervention als auch für die Entwicklung zukünftiger Ansätze abgeleitet werden (Chen 2012). Wie bereits dargestellt, lässt sich das Sprintlernen als didaktisches Rahmenkonzept auf verschiedene Lernthemen, -kontexte und Zielgruppen anpassen, was die Orientierung der Wirkungsevaluation an einem entsprechend umfassenden methodischen Rahmen wie der Programmtheorie erfordert. Dem Konzept des Sprintlernens liegen darüber hinaus mehrere lerntheoretische Ansätze zu Grunde (s. Abschn. 2.2). Es gibt demnach nicht ein bestimmtes theoretisches Konzept oder Modell, das den Gestaltungsansatz vollends begründet und sich damit als durchgängiger Bezugsrahmen für eine ganzheitliche Wirkungsevaluation des Konzepts eignen würde. Aus diesen Gründen wird die theoriegeleitete Evaluation nach Chen als geeigneter methodischer Zugang erachtet, um die in Abschn. 2 dargestellten offenen Fragen zu beantworten.

Im Folgenden werden zunächst die für diesen Beitrag relevanten Begriffe und Hintergründe der Programmevaluation erläutert. Diese werden sodann auf den aktuellen 
Forschungsstand des Sprintlernens übertragen, woraus die Forschungsfragen dieses Beitrags abgeleitet werden.

Nach Chen liegt jeder Intervention eine Theorie zu Grunde, welche im Rahmen der Programmevaluation zu explizieren und zu überprüfen ist. Das Verständnis von Theorie ist dabei sehr breit: Ein „Set an zusammenhängenden Annahmen/Prinzipien und/oder Thesen, das die Aktivitäten einer Intervention erklärt oder leitend dafür ist“" (Chen 1990, S. 40, Übersetzung durch die Autoren).

Unter Programmtheorie wird eine systematische Zusammenstellung der theoretischen Annahmen, die dem Programm zu Grunde liegen, sowie deren Wirkungsweise verstanden. Hierunter können auch implizite Annahmen fallen, die im Rahmen einer programmtheoretisch fundierten Evaluation expliziert werden. Eine vollständige Programmtheorie besteht aus einem Veränderungs- und einem Handlungsmodell - beide werden mit Bezug auf eine bestimmte, $\mathrm{zu}$ evaluierende Intervention aufgestellt und überprüft (Chen 2012).

Das Veränderungsmodell enthält Annahmen über die Wirksamkeit bzw. konkreten Wirkungen eines Programms und wodurch die Effekte generiert werden. Es beinhaltet die Identifizierung und Bestimmung von drei Elementen: Ziele und Outcomes der Intervention, intervenierende Variablen (Wirkmechanismen und kausale Prozesse zwischen Intervention und Outcome) sowie die Intervention selbst.

Das Handlungsmodell beschreibt, wie Kontextfaktoren und die Intervention selbst gestaltet bzw. umgesetzt werden müssen, um die Transformationsprozesse des Veränderungsmodells zu aktivieren. Es beinhaltet präskriptive Annahmen darüber, mit welchen Mitteln und unter welchen Voraussetzungen die Intervention wirkt und umfasst die Beschreibung von Anforderungen an die umsetzende Organisation, die Anwender, den umgebenden Kontext, die Partner, Interventions- und Umsetzungsdokumentationen und die Zielgruppe.

Beide Modelle inkl. ihrer Elemente bilden die übergeordnete Theorie des Programms. Die umfassende theoriegeleitete Evaluation erfordert eine theoretische Spezifizierung aller Elemente und darauffolgende empirische Überprüfungen mit jeweils passenden Evaluationsmethoden. Das systematische Explizieren und Überprüfen der Elemente erfolgt dabei meist nicht gleichzeitig, sondern je nach Forschungsstand und Erkenntnisinteresse in mehreren Untersuchungen (Chen 2012).

Der weiter oben beschriebene Forschungsstand zum Sprintlernen lässt sich vor dem Hintergrund der Programmtheorie wie folgt zusammenfassen. Mit Bezug auf das Handlungsmodell geben die Evaluationsergebnisse (Bauer et al. under review) sowie die Arbeiten zur Qualitätssicherung in der praktischen Umsetzung des Sprintlernens (Jungclaus 2021) Aufschluss darüber, wie die einzelnen Elemente umgesetzt sein müssen, um wirkungs- vollen Kompetenzerwerb, aber auch eine hohe Akzeptanz und Zufriedenheit mit der Lernform des Sprintlernens bei beteiligten Rollenträgern zu erzielen.

Das Veränderungsmodell zum Sprintlernen ist jedoch nur in Teilen expliziert. Die Intervention selbst ist in Form des didaktischen Rahmenkonzepts inkl. Minimalanforderungen zur Umsetzung beschrieben. Es konnte ebenfalls gezeigt werden, dass die Intervention hinsichtlich der Outcomes Kompetenzerwerb sowie Bewertung und Akzeptanz der Lernform wirksam ist. Außerdem wurden die theoretischen Annahmen, die bei der Entwicklung des Sprintlernens handlungsleitend waren, expliziert (s. Abschn. 2.2, lerntheoretische Axiome). Offen ist jedoch, welche intervenierenden Variablen, Wirkmechanismen oder Kausalzusammenhänge potenzielle Bestandteile im Veränderungsmodell des Sprintlernens sind. So gibt es bislang keine hinreichende lerntheoretische Fundierung und durchgängige Betrachtung der theoretischen Konzepte und Bezugspunkte, die den Lernansatz im Besonderen kennzeichnen und zur Erklärung von Wirkprozessen herangezogen werden können.

Vor diesem Hintergrund werden zwei Forschungsfragen formuliert, deren Beantwortung zu einer möglichst umfassenden Explikation der theoretischen Basis der Intervention Sprintlernen und der Erklärung möglicher Transformationsprozesse zwischen Intervention und Outcome führen sollen:

1. Welche spezifischen lerntheoretischen Konstrukte kennzeichnen das Sprintlernen im Besonderen und begründen dessen Wirkweise?

2. Warum sind diese Konstrukte zentral und wichtig zur Erklärung der Wirkprinzipien - was sind also ihre übergeordneten lerntheoretischen Bezugspunkte, aus denen sich potenzielle Wirkmechanismen und kausale Prozesse zur Wirksamkeit des Sprintlernens ableiten lassen?

\section{Methodisches Vorgehen}

Als ersten Schritt zur programmtheoretischen Evaluation einer Intervention und seiner Elemente des Veränderungsmodells benennt Chen die Phase der theoretischen Spezifizierung. Erst, wenn die theoretischen Annahmen der Intervention selbst, mögliche intervenierende Variablen und daran angeschlossene Wirkprozesse expliziert wurden, können diese operationalisiert und im Rahmen weiterer Studien auf Ihre Gültigkeit überprüft werden. Die theoretische Spezifizierung kann unterschiedlich angegangen werden:

- Ableitung aus bestehenden Theorien (gänzlich theoriegeleitet),

- Explikation induktiv beobachteter Wirkmechanismen im Einsatz des Programms,

- Entwicklung mittels Erfahrungsaustausch zwischen Programmentwicklern und/oder Anwendern (Chen 1990). 
Je nach Datenlage kann es sinnvoll sein, mehrere Herangehensweisen zu kombinieren, um die theoretische Basis des Programms möglichst vollständig zu explizieren.

Die theoretische Spezifizierung des Sprintlernens erfolgt durch eine Kombination aus allen drei Vorgehensweisen. Dabei dienten die in Abschn. 3 formulierten Forschungsfragen als konkrete Leitfragen für jeden Analyseschritt der theoretischen Spezifizierung.

In einem ersten Schritt wurden die beiden Leitfragen - d.h. sowohl die Bestimmung der lerntheoretischen Konstrukte, die das Sprintlernen im Besonderen kennzeichnen, als auch deren übergeordneten theoretischen Bezugspunkte - auf Basis induktiv beobachteter Wirkmechanismen im Einsatz des Programms expliziert. Die Praxiserprobungen, die Gegenstand der summativen Evaluationsstudien waren (s. Abschn. 2.3), wurden gezielt aus diesem Blickwinkel betrachtet. Äußerungen, die die Befragten im Rahmen der summativen Evaluation getätigt haben, die Prozessdokumentation aus der Begleitung der Praxisumsetzungen sowie andere umsetzungsbezogene Dokumente zur Ausgestaltung und zum Ablauf der jeweiligen Erprobungen fungierten in diesem Schritt als wichtige Datenquellen. Die Ergebnisse wurden für beide Leitfragen dokumentiert.

Im zweiten Analyseschritt erfolgte eine Ergänzung der theoretischen Spezifizierung aus einem Abgleich mit bestehenden Theorien. Dieser Schritt enthielt zwei Herangehensweisen: Zum einen wurden lerntheoretische Ansätze und Bezugspunkte bestimmt, die sich zur theoretischen Fundierung von Konstrukten des ersten Analyseschritts eignen. Bspw. wurde dem Konstrukt Zielorientierung und Zielverfolgung (Ergebnis aus Analyseschritt 1) die Zielsetzungstheorie als übergeordneter lerntheoretischer Bezugspunkt zugeordnet, weil diese empirisch fundierte Erklärungen zur Wirkweise von Zielen enthält. Zum anderen wurde analysiert, inwieweit die lerntheoretischen Axiome, die handlungsleitend bei der Entwicklung des Sprintlernens waren (s. Abschn. 2.2), zur theoretischen Spezifizierung beitragen können. Mit Blick auf Leitfrage 1 lassen sich aus den Axiomen ggfs. weitere lerntheoretische Konstrukte ableiten - so wurde unter Bezugnahme auf das Axiom zum selbstregulierten Lernen bspw. das Konstrukt Selbstreflexion expliziert und ergänzt. Mit Blick auf Leitfrage 2 können die Axiome zur theoretischen Fundierung bereits identifizierter Konstrukte beitragen - hier wurde bspw. die Selbstbestimmungstheorie der Motivation zur theoretischen Fundierung der Konstrukte Autonomie, Kompetenzerleben und soziale Eingebundenheit herangezogen.

Nach erfolgter Explikation der lerntheoretischen Konstrukte und ihren übergeordneten lerntheoretischen Bezugspunkten, wurden die Ergebnisse zusammengefasst und anhand von schematischen Übersichten visualisiert. Abschließend wurden sie zwischen den drei Programmentwicklerinnen im Rahmen eines Workshops diskutiert und um verein- zelte Aspekte oder Begründungen ergänzt. Alle drei Entwicklerinnen - zwei Psychologinnen, eine Soziologin sind wissenschaftliche Mitarbeiterinnen an anwendungsorientierten Forschungsinstituten und forschen zu arbeitsbezogenem Lernen.

\section{Ergebnisse}

Abb. 2 zeigt die Ergebnisse der theoretischen Spezifizierung im Überblick. Neun verschiedene lerntheoretische Konstrukte kennzeichnen das Sprintlernen im Besonderen und können zur Erklärung von Wirkprinzipien des Gestaltungsansatzes herangezogen werden. Drei der insgesamt sechs übergeordneten Bezugspunkte, die der theoretischen Fundierung der Konstrukte dienen, nehmen dabei Bezug auf die lerntheoretischen Axiome bei der Entwicklung des Sprintlernens: Handlungsorientiertes bzw. selbstreguliertes Lernen, Selbstbestimmungstheorie der Motivation sowie kooperatives Lernen (s.a. Abschn. 4 zur Rolle der lerntheoretischen Axiome bei der theoretischen Spezifizierung). Weitere Bezugspunkte, die im Rahmen der theoretischen Spezifizierung bestimmt wurden, sind die Zielsetzungstheorie (Locke und Latham 2002), die Feedback-InterventionsTheorie (Kluger und DeNisi 1996) und die Theorie der sozialen Interdependenz (Johnson und Johnson 2008).

Im Folgenden werden die einzelnen Konstrukte näher erläutert (s. Forschungsfrage 1). Darüber hinaus wird anhand ihrer lerntheoretischen Bezugspunkte verdeutlicht, aufgrund welcher Mechanismen die Konstrukte die Wirksamkeit des Sprintlernens erklären können (s. Forschungsfrage 2). Ferner wird begründet, weshalb die lerntheoretischen Konstrukte als kennzeichnend für den Gestaltungsansatz erachtet werden, indem auf ihre Umsetzung bzw. Berücksichtigung im Sprintlernen eingegangen wird.

\subsection{Zielorientierung und Zielverfolgung}

Dieses Konstrukt nimmt Bezug auf die Zielgerichtetheit des Handelns bzw. des Handeln-Lernens im Rahmen der Handlungsregulationstheorie. Ziele sind die entscheidenden Orientierungspunkte für die nachfolgenden Prozesse der Handlungssteuerung und damit auch für den Erwerb entsprechender mentaler Präsentationen und Fähigkeiten zur Handlungssteuerung. Zusätzliche Hinweise zur motivierenden und lernförderlichen Gestaltung entsprechender Ziele ergeben sich in diesem Zusammenhang aus der Zielsetzungstheorie. Insbesondere spezifisch und herausfordernd formulierte Lernziele haben einen positiven Einfluss auf die Leistung: Sie lenken Aufmerksamkeit und Anstrengung auf zielrelevante Aktivitäten, erhöhen Energieeinsatz und Ausdauer und führen außerdem dazu, aufgabenrelevantes Wissen und Strategien anzuwenden oder auszubauen. Wei- 
Abb. 2 Lerntheoretische Konstrukte im agilen Sprintlernen mit ihren lerntheoretischen Bezugspunkten

\section{Lerntheoretische Konstrukte im agilen Sprintlernen}

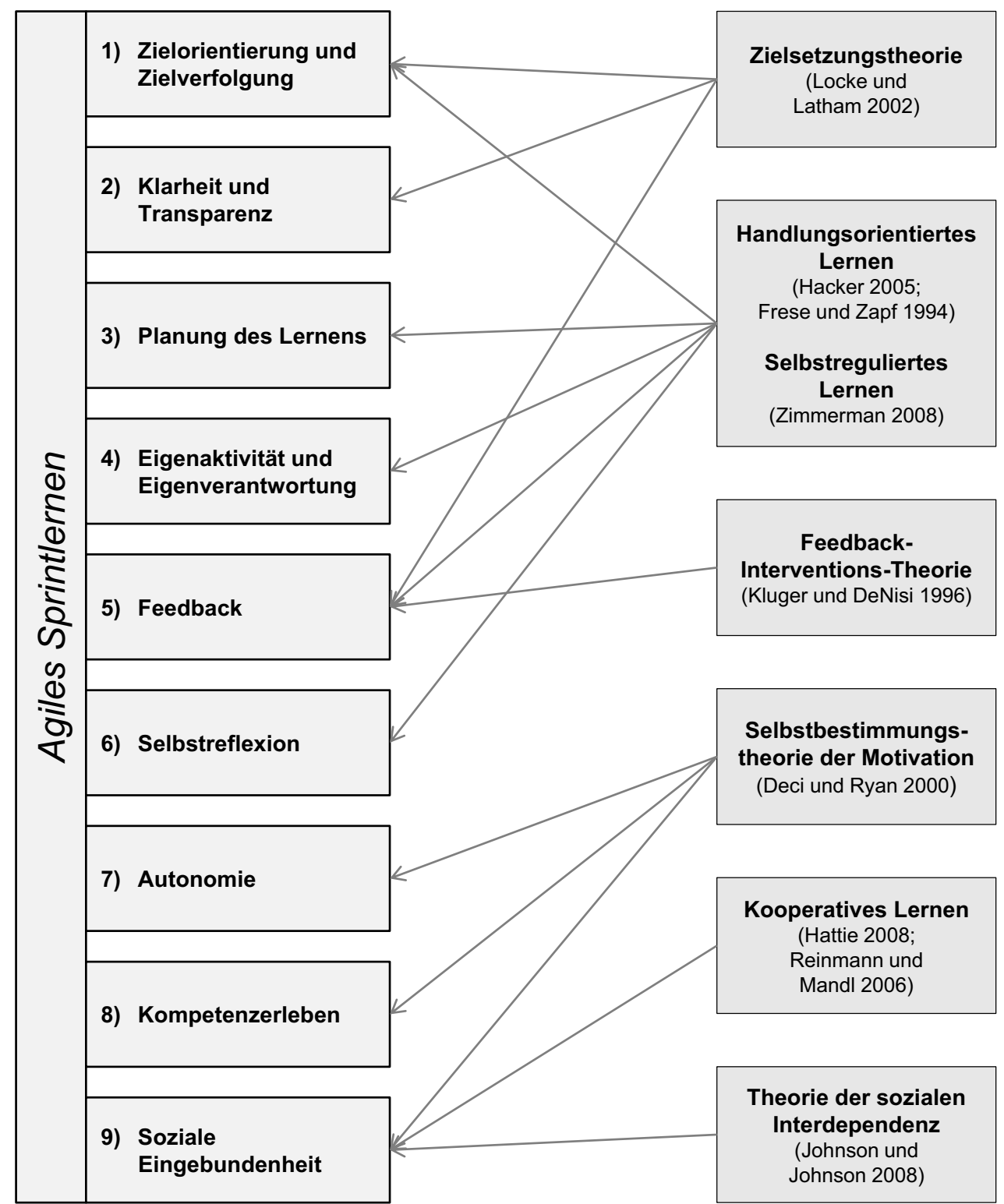

terentwicklungen der Zielsetzungstheorie zeigen darüber hinaus, dass positive Leistungseffekte und wirkungsvolles Lernen vor allem dann resultieren, wenn die Ziele im Sinne einer herausfordernden Lernzielorientierung (d.h. der individuelle und aufgabenbezogen Kompetenzerwerb im Vordergrund steht) und nicht im Sinne einer Leistungszielorientierung (d.h. im Vergleich zu anderen besser zu werden) formuliert werden (Locke und Latham 2002).

Die Orientierung an spezifischen und herausfordernden Lernzielen und deren kontinuierliche Verfolgung spielen im Sprintlernen eine große Rolle. Dies äußert sich in den kontextabhängigen Lernaufgaben mit konkreten Akzeptanzkriterien, welche die Lernziele zur Kompetenzentwick- lung darstellen. Der Status der Erreichung der Ziele wird visualisiert und es gibt feste Feedbackelemente. Durch den iterativen Aufbau mit wiederkehrenden Ergebnisabnahmen wird der Grad der Erfüllung der Lernziele kontinuierlich nachverfolgt und rückgemeldet. Die Lernaufgaben sind im Sinne einer Lernzielorientierung formuliert, weil der Fokus auf den jeweils relevanten Arbeitsaufgaben im beruflichen Handeln der Lernteammitglieder liegt und die dazu benötigten Handlungskompetenzen im Vordergrund stehen. 


\subsection{Klarheit und Transparenz}

Vielfältige Studien und didaktische Ansätze weisen darauf hin, dass Lernprozess und -ergebnis auch davon profitieren, wenn die Lernziele und -gegenstände klar und gut nachvollziehbar präsentiert werden (vgl. z.B. Helmke 2007). Dies beginnt mit der Klarheit und Nachvollziehbarkeit der Lernziele und nimmt damit auf entsprechende Konzepte und Annahmen der Handlungstheorie und der Zielsetzungstheorie Bezug. Insbesondere letzterer Ansatz weist darauf hin, dass durch die Spezifität der Zielformulierung deren Transparenz und Klarheit verbessert wird und damit die Ausrichtung des Handelns eindeutiger gelingt. Weiterhin sollten auch die genauen Lernanforderungen an eine Aufgabe und die Bewertung der Lernziele klar formuliert sein und den Lernenden transparent und nachvollziehbar präsentiert werden (Locke und Latham 2002).

Klarheit und Transparenz der Lernziele und -gegenstände ist auch für das Sprintlernen ein zentrales Gestaltungskriterium, weil Arbeitsprozesse und zugehörige berufliche Handlungskompetenzen in der Vorbereitungsphase transparent herausgearbeitet werden. Die daraus entwickelten Lernaufgaben haben einen sehr hohen Detaillierungsgrad und beinhalten konkrete Akzeptanzkriterien, sodass das Lernteam erkennen kann, was genau erreicht werden soll.

\subsection{Planung des Lernens}

Das Konstrukt Planung ist sowohl im Rahmen des handlungsorientierten als auch im Rahmen des selbstregulierten Lernens von zentraler Bedeutung. Gemäß der Handlungsregulationstheorie (Frese und Zapf 1994; Hacker 2005) folgen auf die Zielbildung die Prozesse Orientierung und Planung, bevor die Handlung ausgeübt wird. Lernende sollen sich bewusst mit der Aufgabe, den Ausführungsmöglichkeiten und den Handlungsbedingungen auseinandersetzen (Orientierung), damit mittels der Planung angemessene und zielführende Strategien für die spezifischen Handlungsanforderungen gewählt werden können (Schaper 2019b). Gemäß den Ansätzen des selbstregulierten Lernens umfasst die Planung eine Aufgabenanalyse und Aspekte der Selbstmotivation. Die Aufgabenanalyse sollte dabei so gestaltet sein, dass sowohl die Zielsetzung als auch eine strategische Planung zur Erreichung des Ziels stattfindet. Selbstregulierte Lerner passen ihre Ziele und die Auswahl ihrer Strategien kontinuierlich an die aktuellen Anforderungen und Randbedingungen an. Relevante motivationale Aspekte der Planung sind u. a. Selbstwirksamkeitsüberzeugungen (d.h. die wahrgenommene Gewissheit, die anstehenden Aufgaben mit den eigenen Fähigkeiten bewältigen zu können), Ergebniserwartung (erwartete Konsequenzen und Folgen der Handlungsausführung) und intrinsisches Interesse (d.h. die Erreichung des Ziels liegt im eigenen Interesse und wird oh- ne äußere Anreize als wichtig erachtet). Eine hohe Ausprägung dieser Aspekte führt zu höheren Lernleistungen. Positive Lernerfahrungen (z. B. Zufriedenheit mit dem Lernerfolg) aus vorangegangenen Lernphasen zu einem Thema können sich positiv auf diese motivationalen Aspekte der Planungsphase und damit wiederum auf die nachfolgende Leistung auswirken (Zimmerman 2008).

Im Sprintlernen wird das Konstrukt Planung durch das explizite Planungstreffen vor jedem Lernsprint umgesetzt. Die Gestaltung des Planungstreffens berücksichtigt beide Prozesse, die im Sinne des handlungsorientierten Lernens relevant sind: Aufgabenanalyse und Selbstmotivation. Die Lernteams setzen sich mit den anstehenden Lernaufgaben auseinander. Bei Bedarf stellen sie inhaltliche Rückfragen oder Fragen zur Ergebniserwartung an den Fachexperten, sofern sich dies nicht eindeutig aus den Lernaufgaben und Akzeptanzkriterien ergibt. Darüber hinaus planen sie aufgabenabhängig, mit welcher Strategie sie die Lernaufgaben im kommenden Lernsprint angehen wollen. Erkenntnisse aus der Prozessreflexion des vorangegangenen Lernsprints werden dabei explizit berücksichtigt. Die Lernaufgaben stehen in Zusammenhang und tragen zu einem transparenten übergeordneten Ziel bei, das für den beruflichen Alltag der Lernenden von Relevanz ist.

\subsection{Eigenaktivität und Eigenverantwortung}

Dieses Konstrukt bezieht sich auf die Prozesse der Handlungsausführung und -kontrolle des handlungsorientierten Lernens (Frese und Zapf 1994; Hacker 2005) und die Lernund Leistungsphase im selbstregulierten Lernen (Zimmerman 2008). Während der Handlung setzen sich die Lernenden aktiv und kognitiv-reflektierend mit ihrem Vorgehen auseinander. Der Lernende wendet eigene Strategien der Ausführung an und ist aktiv handelnd. Er kontrolliert und beobachtet sich selbst hinsichtlich der Zielerreichung und der angewandten Strategien und muss seine Aufmerksamkeit auf sein Handeln bzw. die Aufgabe und den jeweils nächsten relevanten Schritt zur Zielerreichung fokussieren können. Das Sprintlernen verlangt den Lernenden daher ein hohes $\mathrm{Maß}$ an Eigenaktivität und Eigenverantwortung ab. So wendet das Lernteam die eigens geplanten Strategien während der selbstgestalteten Lernzeit an und passt diese bei Bedarf eigenverantwortlich an. Fachexperten und Sprintbegleiter unterstützen in dieser Phase nur auf Anfrage. Die präzise formulierten Lernaufgaben erleichtern die Fokussierung der Aufmerksamkeit und Lernende können sich mittels der Akzeptanzkriterien zur Zielerreichung bewusst selbst kontrollieren und ihr Vorgehen adaptieren. 


\subsection{Feedback}

Feedback zu den Lernzielen verstärkt die Lernanstrengungen Im Sinne der Zielsetzungstheorie (Locke und Latham 2002) und vermittelt Informationen über das Ausmaß der Zielerreichung im Sinne der Handlungsregulationstheorie (Frese und Zapf 1994; Hacker 2005). Es wirkt sich jedoch nicht per se motivations- oder leistungsfördernd aus. Gemäß der Feedback-Interventions-Theorie (Kluger und DeNisi 1996) ist die Art der Rückmeldung ein wesentlicher Faktor für die Auswirkungen auf die Leistung. Feedback kann auf unterschiedlichen Ebenen erfolgen: Je nach Art und Inhalt des Feedbacks wird die Aufmerksamkeit auf Prozesse der Meta-Aufgabenebene (inkl. selbstbezogene Aspekte), der Aufgaben-Motivationsebene oder der Aufgaben-Lernebene gelenkt. Je mehr das Feedback auf die Aufgaben-Lernebene gerichtet ist, desto vorteilhafter wirkt sich dies auf die Lernleistung aus. Darüber hinaus begünstigt regelmäßiges und häufiges Feedback positive Lernbzw. Leistungseffekte (Kluger und DeNisi 1996).

Der Lernprozess im Sprintlernen ist stark durch Feedback gekennzeichnet. Während der selbstgestalteten Lernzeit hat das Lernteam durch die Akzeptanzkriterien die Möglichkeit zur Selbstkontrolle, weil es die Soll-Anforderungen der Lernaufgaben mit dem Ist-Stand abgleichen kann und sich auf diese Weise selbst Feedback gibt. In den Ergebnisabnahmen erhält das Lernteam nach dem Lernsprint Feedback der Fachexperten - ebenfalls anhand der zuvor definierten Akzeptanzkriterien. Die Fachexperten gehen dabei konkret auf Diskrepanzen zwischen Soll und Ist ein, d.h. die Rückmeldung bezieht sich primär auf die Aufgabenausführung und weniger auf die Personen selbst. Das Feedback erfolgt demnach vor allem auf der AufgabenLernebene, was bedeutsam für positive Auswirkungen von Feedback auf die Leistung ist.

\subsection{Selbstreflexion}

Dieses Konstrukt nimmt Bezug auf die Prozesse des Feedbacks im handlungsorientierten Lernen (Frese und Zapf 1994; Hacker 2005) und die Selbstreflexionsphase im selbstregulierten Lernen (Zimmerman 2008). Die Selbstreflexion ist ein zentraler Prozess zum Abschluss der Handlung. Im Rahmen der Selbstbewertung vergleichen Lernende ihr Ergebnis mit dem angestrebten Ziel und suchen Begründungen für das Erreichen bzw. Nicht-Erreichen des Ziels. Auch Emotionen, die durch die Lernhandlung und das Ergebnis ausgelöst werden, sind hier von Bedeutung. Alle Bewertungskomponenten liefern wertvolle und handlungsspezifische Informationen zum zurückliegenden Lernprozess, die in den nächsten Handlungszyklus in Form von Anpassungen oder einem Beibehalten bewährter Strategien einfließen (Schmitz und Wiese 2006).
Neben etwaigen Anregungen zur Selbstreflexion durch die Selbstkontrolle anhand der Akzeptanzkriterien in der selbstgestalteten Lernzeit endet jeder Lernsprint im Sprintlernen mit zwei Feedbackeinheiten. In der Ergebnisabnahme erhält das Lernteam Feedback durch die Fachexperten, bevor in der Prozessreflexion explizit der Lernprozess reflektiert wird. In diesem Ablaufelement hat auch der reflektierte Umgang mit Emotionen des Lernteams Raum. Die eingesetzten Strategien werden umfassend bewertet und das Lernteam leitet Maßnahmen für den nächsten Lernsprint ab. Durch die Portionierung der Lerninhalte und den iterativen Aufbau werden diese Soll-Ist-Abgleiche regelmäßig vorgenommen und Anpassungen können zeitnah umgesetzt und erneut reflektiert werden.

\subsection{Lernförderliche psychische Bedürfnisse der Selbstbestimmungstheorie}

Die Selbstbestimmungstheorie der Motivation (Deci und Ryan 2000) postuliert, dass drei psychologische Grundbedürfnisse für Motivation relevant sind: Das Bedürfnis nach Autonomie, Kompetenz und sozialer Eingebundenheit. Das Vorhandensein dieser Faktoren begünstigt das Auftreten intrinsischer bzw. die Integration extrinsischer Motivation im Lernen.

Autonomie heißt, dass Lernende eigene Entscheidungsspielräume haben und selbstbestimmt handeln können z.B. in Bezug auf Lerninhalte und Lernstrategien. Im Sprintlernen äußert sich Autonomie vor allem in der eigenständigen Planung der Lernstrategien und Lernzeiten sowie einem hohen Grad an Selbstbestimmung während der selbstgestalteten Lernzeit. Geringe Entscheidungsspielräume gibt es wiederum bei der Auswahl und Definition der Ziele. Die Lerngegenstände ergeben sich direkt aus dem Arbeitskontext bzw. -prozess. Sie werden entweder unmittelbar durch den Kunden beauftragt oder durch ihn „freigegeben“, falls der Kompetenzbedarf durch die Mitarbeitenden selbst geäußert wurde. Die konkreten Lernziele und -aufgaben werden vorab vom Fachexperten definiert, wobei das Lernteam Änderungen oder Ergänzungen anregen kann, die während des Lernens in Abstimmung mit dem Fachexperten umgesetzt werden.

Kompetenzerleben als psychisches Grundbedürfnis beschreibt, dass Lernende im Lernprozess Erfolgserlebnisse haben sollten, die die eigene Handlungsfähigkeit in Bezug auf aktuelle und zukünftige Herausforderungen zeigt und sich dadurch motivierend auf den weiteren Lernprozess auswirken (Deci und Ryan 2000). Das Auftreten von Kompetenzerleben wird im Sprintlernen durch die hohe Eigenverantwortung in der selbstgestalteten Lernzeit, die Visualisierung des Lernfortschritts sowie den iterativen Ablauf mit wiederkehrenden Erfolgsmomenten in den Ergebnisabnahmen begünstigt. Ein positives Kompetenzerleben ist 
allerdings im Rahmen dieser Lernprozesse nicht garantiert: Bei den Ergebnisabnahmen können durchaus auch Misserfolge erlebt werden, was unter Umständen dazu führt, dass die jeweiligen Lernaufgaben im nächsten Lernsprint erneut bearbeitet werden müssen.

Das Konstrukt soziale Eingebundenheit beinhaltet gemäß der Selbstbestimmungstheorie sowohl die Zugehörigkeit zu einer sozialen Gruppe als auch soziale Akzeptanz. Das heißt, dass Lernende ein Gefühl der Zugehörigkeit und Verbundenheit haben sollten - z. B. mit anderen Lernenden oder Lehrpersonen. Dazu zählen auch die wahrgenommene soziale Unterstützung und eine positiv geprägte und sichere Bindung im Sinne eines ,sich akzeptiert-Fühlens“ und einer respektvollen und rücksichtsvollen Interaktion im sozialen Gefüge (Deci und Ryan 2004).

Im Sprintlernen sind Lernende in ihr Lernteam eingebunden, womit das Merkmal der sozialen Eingebundenheit realisiert wird. Lernteam, Sprintbegleiter und Fachexperten bilden ebenfalls eine soziale Gruppe und rahmen damit zusätzlich die soziale Eingebundenheit im Lernteam. In der Prozessreflexion werden Lernprozess und Zusammenarbeit des Lernteams explizit durch den Sprintbegleiter thematisiert und gemeinsam reflektiert. Bei Bedarf werden Anpassungen für den nächsten Lernsprint abgeleitet. Im Sinne der Interdependenztheorie (Johnson und Johnson 2008) liegt eine positive Interdependenz der Ziele im kooperativen Lernen vor, weil das Ziel eines einzelnen Lernteammitglieds dann erreicht ist, wenn die anderen Lerner das Ziel ebenfalls erreichen. Bestimmte Lernaufgaben sind so gestaltet, dass das Ziel nur durch gemeinsames Handeln erreicht wird - z.B., wenn verschiedene Berufsgruppen an einem Arbeitsprozess beteiligt sind und sie sich in ihrem Wissen für eine Lernaufgabe ergänzen. Dies führt zu lernförderlicher Kooperation und gegenseitiger Unterstützung. Positive Interdependenzen und das daraus resultierende kooperative Verhalten der Gruppenmitglieder sind für positive Folgen auf den Lernprozess entscheidend. Im Vergleich zu kompetitivem sowie individualistischem Verhalten führt kooperatives Verhalten u.a. zu besseren Lernergebnissen, besserer Erinnerungsleistung und höher ausgeprägtem kritischen Denken.

\section{Diskussion}

Im Folgenden werden die Ergebnisse zusammengefasst, auf Limitationen hingewiesen und wissenschaftliche wie praktische Implikationen abgeleitet.

Im Rahmen der vorgestellten theoretischen Spezifizierung wurden neun lerntheoretische Konstrukte identifiziert, die die Gestaltung des Sprintlernens im Besonderen kennzeichnen: Zielorientierung und Zielverfolgung, Klarheit und Transparenz, Planung, Eigenaktivität und Eigenverant- wortung, Feedback, Selbstreflexion, Autonomie, Kompetenzerleben und soziale Eingebundenheit. Die Konstrukte beschreiben die Intervention Sprintlernen aus einer lerntheoretischen Perspektive. Im Sinne von Chen (Chen 1990, 2012) sind sie Bestandteile im Veränderungsmodell des Lernansatzes, weil sie verschiedene Wirkmechanismen zum Tragen bringen und damit zur Erklärung der Wirkweise des Sprintlernens beitragen können. Für jedes Konstrukt wurde anhand übergeordneter theoretischer Bezugspunkte beleuchtet, welche konkreten Wirkprinzipien Teil des Veränderungsmodells sind und wie der Kompetenzzugewinn zu Stande kommen kann.

Wie bereits erwähnt, wird das Konstrukt Autonomie nicht in vollumfänglicher Ausprägung (im Sinne der Selbstbestimmungstheorie) im Sprintlernen umgesetzt. Dadurch, dass es sich um einen Ansatz für arbeitsbezogene Kompetenzentwicklung handelt, beinhaltet die grundlegende Ausrichtung des Sprintlernens eine Art Auftraggeber-Umsetzer-Verhältnis (s. Abschn. 2.1.1). Dieses führt dazu, dass die Lernenden zwar große Handlungsspielräume im Lernprozess, aber begrenzte Entscheidungsspielräume bei der Auswahl von Lerngegenständen haben (s. Abschn. 2.1.1 und 5, Autonomie). Es ist daher denkbar, dass Lernteammitglieder die Lernziele und -inhalte als fremdbestimmt erleben - auch, wenn die Relevanz des Lernthemas für das Lernteam ein wichtiges Kriterium für die Auswahl und Eignung der Lernthemen im Sprintlernen darstellt. Daraus könnte resultieren, dass die dargestellten Wirkprinzipien und positiven Effekte von Autonomie nicht oder nur eingeschränkt zum Tragen kommen.

Durch die vorliegende Arbeit wurden die theoretischen Annahmen und potenziellen Elemente des Veränderungsmodells expliziert. Dies kann jedoch lediglich der Ausgangspunkt einer umfassenden Evaluation sein, die (empirisch gesichert) Aufschluss über die tatsächliche Wirkweise des Sprintlernens in der Praxis gibt. Darüber hinaus sollte auch die theoretische Spezifizierung selbst auf Gültigkeit überprüft werden. Die vorliegenden Ergebnisse beschränken sich auf beobachtete und induktiv herausgearbeitete Wirkmechanismen, den Abgleich mit bestehenden Theorien und einen Erfahrungsaustausch der Programmentwicklerinnen - die Perspektive von Anwendern in der Praxis fehlte u.E. Sind die Konstrukte auch aus Sicht von beteiligten Rollenträgern in der Umsetzung des Sprintlernens vorhanden? Werden sie als lernförderlich erachtet? Sind möglicherweise weitere Konstrukte kennzeichnend für den Gestaltungansatz? Diese Fragen gilt es in einem nächsten Untersuchungsschritt empirisch zu überprüfen. Dies ist auch bereits erfolgt und soll in einem weiteren Beitrag der Autoren vorgestellt werden (Jungclaus und Schaper under review).

Darüber hinaus ist zu beachten, dass bestimmte Wirkprinzipien im Sprintlernen durch die spezifische Kombina- 
tion oder ein Zusammenspiel der neun lerntheoretischen Konstrukte ausgelöst oder verstärkt werden können. So könnte der erfolgreiche Kompetenzerwerb bspw. in der Kombination aus Zielorientierung/-verfolgung mit aufgabenbezogenem Feedback begründet sein, weil Zielorientierung zu erhöhten Leistungen und aufgabenbezogenes Feedback zu entsprechenden Leistungsanstrengungen führt und motivationsförderlich ist (Kluger und DeNisi 1996; Locke und Latham 2002). Ebenso könnte sich die explizite Berücksichtigung und Kombination der Konstrukte Planung, Eigenaktivität, Feedback und Selbstreflexion im Sinne der Handlungsregulationstheorie positiv auf den Lernerfolg auswirken, weil alle Phasen des Handeln-Lernens berücksichtigt werden und sich das Lernteam aktiv und kognitivreflektierend mit den Handlungsanforderungen einer Aufgabe bzw. Tätigkeit auseinandersetzt. Dadurch entwickeln die Lernenden vollständige und effektive mentale Repräsentationen zur Handlungssteuerung im jeweiligen Lernthema. Die iterative Struktur des Sprintlernens beinhaltet Feedback und Selbstreflexion in bzw. nach jedem Lernsprint, welches in den nächsten Planungs- und aktiven Handlungsphasen aufgegriffen wird. Die stetigen Soll-Ist-Abgleiche ermöglichen eine kontinuierliche Anpassung der Handlungen und des Lernverhaltens.

Ggfs. werden durch das Sprintlernen jedoch auch benötigte Kompetenzen für selbstreguliertes Lernen gefördert, sodass die Leistung der Lernenden durch die Befähigung zum selbstregulierten Lernen steigt. Es gilt, diese exemplarisch angedeuteten Wechselwirkungen im Rahmen weiterführender Forschung systematisch im Blick zu behalten, um $\mathrm{zu}$ evaluieren, warum und wie genau das Sprintlernen tatsächlich wirksam ist. Dazu zählt auch, die Wirkweise und das Einflussgewicht der einzelnen lerntheoretischen Konstrukte differenziert zu betrachten.

Aus diesem Aspekt lässt sich bereits eine Implikation für die Praxis ableiten: Die Ergebnisse der theoretischen Fundierung untermauern die Wichtigkeit der hochwertigen Umsetzung und Ausgestaltung des didaktischen Rahmenkonzepts agiles Sprintlernen in der Praxis. Werden wirkungsrelevante Gestaltungselemente nicht oder nicht ,gut genug" umgesetzt, kann dies die Wirkung beeinträchtigen. So ist neben bereits bekannten Aspekten zur Gestaltung einer Ergebnisabnahme bspw. auch darauf zu achten, dass die Fachexperten ihr Feedback konsequent auf der Aufgabenebene geben. Die Qualität der Lernaufgaben - insbes. die Formulierung der Ziele (z.B. Ansprüche an Spezifität und Messbarkeit) - ist ein weiteres Beispiel für umsetzungsrelevante Konsequenzen, die sich aus den vorliegenden Ergebnissen ergeben.

Vor diesem Hintergrund kann es ratsam sein, die wichtigsten Aspekte der theoretischen Fundierung in das Qualifizierungskonzept für Sprintbegleiter aufzunehmen (siehe Jungclaus et al. 2021). Dadurch könnten angehende Sprint- begleiter aus der Praxis neben umsetzungsbezogenen Kompetenzen auch ein lerntheoretisches Hintergrundwissen aufbauen, um die Bedeutung der lerntheoretischen Konstrukte und deren Wirkweise tiefgründiger zu erfassen und bei ihrem Handeln bzw. Eingreifen in Prozesse des Lernteams zu berücksichtigen.

Hinsichtlich der Entwicklung von neuartigen Ansätzen zur arbeitsbezogenen Kompetenzentwicklung lässt sich sagen, dass sich die Kombination der verschiedenen lerntheoretischen Axiome (s. Abschn. 2.2) und das Zusammenspiel unterschiedlicher lerntheoretischer Ansätze im Sprintlernen bewährt haben. Auch zukünftige Ansätze sollten nicht ,nur“ hinsichtlich ihrer Wirksamkeit, sondern umfassend im Sinne einer Explikation der zu Grunde liegenden theoretischen Annahmen und potenziellen Wirkmechanismen evaluiert werden. Das trägt dazu bei, die Relevanz und Wirksamkeit von theoretischen Konstrukten und Ansätzen in der Praxis besser zu verstehen und zu gestalten.

Funding Open Access funding enabled and organized by Projekt DEAL.

Open Access Dieser Artikel wird unter der Creative Commons Namensnennung 4.0 International Lizenz veröffentlicht, welche die Nutzung, Vervielfältigung, Bearbeitung, Verbreitung und Wiedergabe in jeglichem Medium und Format erlaubt, sofern Sie den/die ursprünglichen Autor(en) und die Quelle ordnungsgemäß nennen, einen Link zur Creative Commons Lizenz beifügen und angeben, ob Änderungen vorgenommen wurden.

Die in diesem Artikel enthaltenen Bilder und sonstiges Drittmaterial unterliegen ebenfalls der genannten Creative Commons Lizenz, sofern sich aus der Abbildungslegende nichts anderes ergibt. Sofern das betreffende Material nicht unter der genannten Creative Commons Lizenz steht und die betreffende Handlung nicht nach gesetzlichen Vorschriften erlaubt ist, ist für die oben aufgeführten Weiterverwendungen des Materials die Einwilligung des jeweiligen Rechteinhabers einzuholen.

Weitere Details zur Lizenz entnehmen Sie bitte der Lizenzinformation auf http://creativecommons.org/licenses/by/4.0/deed.de.

\section{Literatur}

von Ameln, F., \& Wimmer, R. (2016). Neue Arbeitswelt, Führung und organisationaler Wandel. Gruppe. Interaktion. Organisation, 47(1), 11-21.

Bauer, A., Jungclaus, J., \& Arndt, P. A. (under review). Arbeitsbezogene Kompetenzen erfolgreich entwickeln. Evaluation des agilen Sprintlernens in der Praxis. Zeitschrift für Arbeits- und Organisationspsychologie.

Chen, H.-T. (1990). Theory-driven evaluations. California: SAGE.

Chen, H.-T. (2012). Theory-driven evaluation: Conceptual framework, application and advancement. In R. Strobl, O. Lobermeier \& W. Heitmeyer (Hrsg.), Evaluation von Programmen und Projekten für eine demokratische Kultur. Wiesbaden: Springer.

Deci, E. L., \& Ryan, R. M. (2000). The "what" and "why" of goal pursuits: human needs and the self-determination of behavior. Psychological Inquiry, 11(4), 227-268.

Deci, E. L., \& Ryan, R. M. (2004). Handbook of self-determination research. Rochester, NY: University of Rochester Press.

Frese, M., \& Zapf, D. (1994). Action as the core of work psychology: a German approach. In C. Triandis, M. D. Dunnette \& L. M. Hough 
(Hrsg.), Handbook of industrial and organizational psychology (S. 271-340). Palo Alto: Consulting Psychologists Press.

Hacker, W. (2005). Allgemeine Arbeitspsychologie. Bern: Huber.

Hattie, J. (2008). Visible learning: a synthesis of over 800 meta-analyses relating to achievement. London: Routledge.

Helmke, A. (2007). Lernprozesse anregen und steuern. Was wissen wir über Klarheit und Strukturiertheit? Pädagogik, 59(6), 44-47.

Johnson, D. W., \& Johnson, R. T. (2008). Social interdependence theory and cooperative learning: the teacher's role. In R.M. Gillies, A.F. Ashman \& J. Terwel (Hrsg.), The teacher's role in implementing cooperative learning in the classroom. New York: Springer.

Jungclaus, J. (2021). Worauf kommt es an? Qualitätssicherung im agilen Lernen. In J. Longmuss, G. Korge, A. Bauer \& B. Höhne (Hrsg.), Agiles Lernen im Unternehmen (S. 111-118). Wiesbaden: Springer Vieweg.

Jungclaus, J., \& Schaper, N. (under review). Theorie versus Praxis: Lerntheoretische Spezifizierung des Lernkonzepts „Agiles Sprintlernen“ auf dem Prüfstand. Journal of Technical Education (JOTED).

Jungclaus, J., Korge, G., Arndt, P., \& Bauer, A. (2019). Agiles Sprintlernen - ein Konzept für dezentrales betriebliches Lernen: Empirische Begründung und praktische Erfahrungen. Gruppe. Interaktion. Organisation. Zeitschrift für Angewandte Organisationspsychologie (GIO), 50(2), 217-227.

Jungclaus, J., Arndt, P. A., \& Bauer, A. (2021). Begleitung will gelernt sein - ein Qualifizierungskonzept für die Rolle der methodischen Begleitung. In J. Longmuss, G. Korge, A. Bauer \& B. Höhne (Hrsg.), Agiles Lernen im Unternehmen (S. 119-126). Wiesbaden: Springer Vieweg.

Kirschner, P. A., Sweller, J., \& Clark, R.E. (2006). Why minimal guidance during instruction does not work: an analysis of the failure of Constructivist, discovery, problem-based, experiential, and inquiry-based teaching. Educational psychologist, 41(2), 75-86.

Kluger, A. N., \& DeNisi, A. (1996). The effects of feedback interventions on performance: a historical review, a meta-analysis, and a preliminary feedback intervention theory. Psychological Bulletin, 119(2), 254-284.

Korge, G., Jungclaus, J., \& Bauer, A. (2018). Agiles Sprintlernen. Eine neue Lernform für die digitalisierte Arbeitswelt. Zeitschrift für wirtschaftlichen Fabrikbetrieb, 113(10), 637-640.

Locke, E. A., \& Latham, G.P. (2002). Building a practically useful theory of goal setting and task motivation: a 35-year odyssey. American psychologist, 57(9), 705-717.

Mietzel, G. (2017). Pädagogische Psychologie des Lernens und Lehrens. Göttingen: Hogrefe.

Rauner, F., Grollmann, P., \& Martens, T. (2007). Messen beruflicher Kompetenz(entwicklung). ITS-Forschungsberichte, Bd. 21. Bremen: Institut Technik und Bildung.

Reinmann, G., \& Mandl, H. (2006). Unterrichten und Lernumgebungen gestalten. In A. Krapp \& B. Weidenmann (Hrsg.), Pädagogische Psychologie. Ein Lehrbuch (S. 613-658). Weinheim: Beltz.

Roock, S., \& Wolf, H. (2015). Scrum - verstehen und erfolgreich einsetzen. Heidelberg: dpunkt.

Schaper, N. (2000). Gestaltung und Evaluation arbeitsbezogener Lernumgebungen. Habilitationsschrift. Heidelberg: Ruprecht-KarlsUniversität Heidelberg.

Schaper, N. (2007). Lerntheorien. In H. Schuler \& K. Sonntag (Hrsg.), Handbuch der Arbeits- und Organisationspsychologie (S. 43-50). Göttingen: Hogrefe.

Schaper, N. (2019a). Aus- und Weiterbildung: Konzepte der Trainingsforschung. In F.W. Nerdinger, G. Blickle \& N. Schaper (Hrsg.), Arbeits- und Organisationspsychologie (S. 509-539). Berlin: Springer.
Schaper, N. (2019b). Theoretische Modelle des Arbeitshandelns. In F.W. Nerdinger, G. Blickle \& N. Schaper (Hrsg.), Arbeits- und Organisationspsychologie (S. 359-383). Berlin: Springer.

Schaper, N., \& Sonntag, K. (2007). Weiterbildungsverhalten. In D. Frey \& L. von Rosenstiel (Hrsg.), Wirtschaftspsychologie Bd. 1. Göttingen: Hogrefe.

Schmitz, B., \& Wiese, B. S. (2006). New perspectives for the evaluation of training sessions in self-regulated learning: time-series analyses of diary data. Contemporary Educational Psychology, 31(1), 64-96.

Zimmerman, B.J. (2008). Investigating self-regulation and motivation: historical background, methodological developments, and future prospects. American Educational Research Journal, 45(1), 163-183.

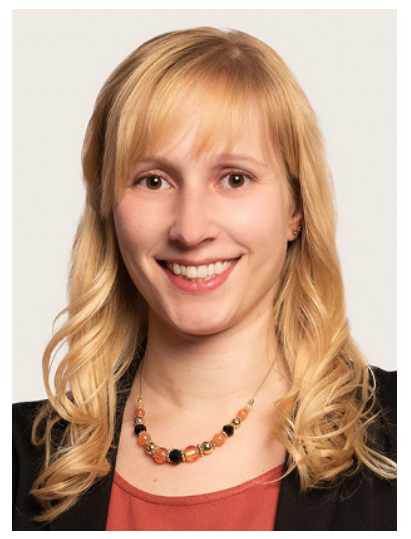

Joana Jungclaus ist wissenschaftliche Mitarbeiterin und Mitglied des Leitungsteams am ZNL TransferZentrum für Neurowissenschaften und Lernen der Universität Ulm. Sie ist Wirtschaftspsychologin und ihre Forschungsschwerpunkte und -interessen liegen in den Bereichen Kompetenzmanagement, organisationales Lernen, Organisationsentwicklung, Wissensmanagement und Coaching. $\mathrm{Zu}$ ihren aktuellen Schwerpunkten gehören u. a. selbstgesteuerte Lernformen und deren Effekte auf Kompetenzentwicklung und organisationales Lernen.

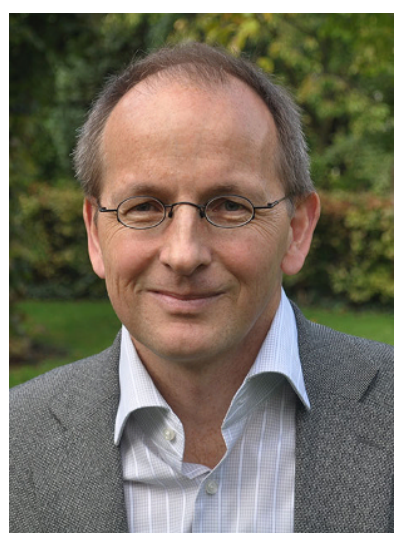

Prof. Dr. Niclas Schaper ist Professor für Arbeits- und Organisationspsychologie an der Universität Paderborn. Er hat verschiedene Forschungsprojekte zu Fragen des Kompetenzmanagements bei der strategischen Personalarbeit in KMUs und bei der Curriculum- und Personalentwicklung im Hochschulkontext geleitet. AuBerdem war er mit verschiedenen Teilprojekten an der Clusterförderung IT'sOWL zu Fragen von Industrie 4.0 und Arbeit 4.0 beteiligt. Aktuell beschäftigt er sich in einem BMBF-Projekt im Kontext der Förderlinie „Zukunft der Arbeit“ mit Fragen der partizipativen und humangerechten Begleitung von KMUs bei der digitalen Transformation von Arbeitsprozessen. Seine Forschungsschwerpunkte und -interessen liegen in den Bereichen Kompetenzerfassung und -modellierung, betriebliche Laufbahngestaltung, betriebliches Weiterbildungsverhalten insbesondere in Form von informellen Lernaktivitäten im Arbeitskontext und seine Einflussfaktoren, Erfassung organisationaler Lernkulturen, Weiterentwicklung von Arbeits- und Anforderungsanalysen, Mitarbeiterbefragungen und organisationale Bindung von Mitarbeitern. 